\title{
FRSMC DESIGN FOR THE STEERING CONTROL AND DIVING CONTROL OF UNDERWATER VEHICLES
}

\author{
Tzu-Chun Kuo \\ Department of Electrical Engineering, Ching Yun University, Chungli, Taiwan, R.O.C., tck@mail.cyu.edu.tw \\ Ying-Jeh Huang \\ Department of Electrical Engineering, Yuan Ze University, Chungli, Taiwan, R.O.C. \\ Hung-Hsiu Yu \\ Robot Control Technology Department, Mechanical and Systems Research Laboratories, Industrial Technology \\ Research Institute, Hsinchu, Taiwan, R.O.C.
}

Follow this and additional works at: https://jmstt.ntou.edu.tw/journal

Part of the Electrical and Computer Engineering Commons

\section{Recommended Citation}

Kuo, Tzu-Chun; Huang, Ying-Jeh; and Yu, Hung-Hsiu (2009) "FRSMC DESIGN FOR THE STEERING CONTROL AND DIVING CONTROL OF UNDERWATER VEHICLES," Journal of Marine Science and Technology. Vol. 17: Iss. 1, Article 8. DOI: $10.51400 / 2709-6998.1977$

Available at: https://jmstt.ntou.edu.tw/journal/vol17/iss1/8

This Research Article is brought to you for free and open access by Journal of Marine Science and Technology. It has been accepted for inclusion in Journal of Marine Science and Technology by an authorized editor of Journal of Marine Science and Technology. 
FRSMC DESIGN FOR THE STEERING CONTROL AND DIVING CONTROL OF UNDERWATER VEHICLES

Acknowledgements

The authors would like to thank the National Science Council, Taiwan, R.O.C., for supporting this work under Grant NSC95-2221-E-231-013 and NSC95-2622-E-155-008-CC3. 


\title{
FRSMC DESIGN FOR THE STEERING CONTROL AND DIVING CONTROL OF UNDERWATER VEHICLES
}

\author{
Tzu-Chun Kuo*, Ying-Jeh Huang**, and Hung-Hsiu Yu***
}

Key words: autonomous underwater vehicle, position control, uncertain systems, robustness.

\begin{abstract}
A robust control method is proposed for the steering and diving control systems of autonomous underwater vehicles. Heading angle and heave position tracking are achieved. All the closed-loop poles in the sliding mode can be placed by using the sliding mode and root locus techniques. Fuzzy logic technique is further employed to effectively tune the control law. Parameter variation and external disturbance such as various vehicle speeds and the ocean current effect are overcome.
\end{abstract}

\section{INTRODUCTION}

In recent years, underwater vehicles have been widely used for inspecting the ocean. Underwater operations have become deeper and underwater operation tasks have become more difficult and dangerous beyond what divers can possibly sustain. While operating an unmanned underwater vehicle, correct positioning is important so that the vehicle can move along the desired path as expected $[4,16]$. Thus, equipped with a good automatic control system, operators can then concentrate on their work without having to worry about the position control.

Unmanned underwater vehicles are difficult to control and can easily be affected during sailing by un-expected ocean current. Various theories for the control of underwater vehicles have been proposed in recent years such as intelligent control, sliding mode control, adaptive control, and so on $[2,3,5,8,10$, $14,18,20]$. In [2], the authors proposed an adaptive network structure for a multivariable Sugeno style fuzzy inference system to solve the problem of autonomous underwater vehicle autopilot design. This method compared with a traditional multi-input single-output control approach whereby control of

Paper submitted 10/18/07; accepted 02/25/08. Author for correspondence: Tzu-ChunKuo (e-mail: tck@mail.cyu.edu.tw).

*Department of Electrical Engineering, Ching Yun University, Chungli, Taiwan, R.O.C.

**Department of Electrical Engineering, Yuan Ze University, Chungli, Taiwan, R.O.C.

**Robot Control Technology Department, Mechanical and Systems Research Laboratories, Industrial Technology Research Institute, Hsinchu, Taiwan, R.O.C. each degree of freedom was considered separately. No proof was made for solving the inherent cross-coupling between autonomous underwater vehicle channel motions. In [10], a one-layer neural-network controller with preprocessed input signals was designed to control the vehicle track along the desired position and attitude with the absence of unknown disturbances and modeling errors. The paper presented the utilization of a self-adaptive recurrent neuro-fuzzy control as a feedforward controller and a proportional-plus-derivative control as a feedback controller for controlling an autonomous underwater vehicle in an unstructured environment [18]. The PD feedback controller computed the error torque to minimize the system error along the desired trajectory. A systematic self-adaptive learning algorithm, consisting of a mappingconstrained agglomerative clustering algorithm for the structure learning and a recursive recurrent learning algorithm for the parameter learning, was developed to construct the recurrent neuro-fuzzy system to model the inverse dynamics of an autonomous underwater vehicle with fast learning convergence.

To deal with the parameter variation and external disturbance, sliding mode control (SMC) has attracted a great deal of attention $[4,9,14,15,19]$. In the sliding mode, the SMC system performs like an equivalent lower-dimensional system. Placing all of the closed-loop poles is not easy. Some closed-loop poles must inherently be located at the origin [6]. Control system responses in the sliding mode, such as transient and steady state responses, could not be easily specified.

In this paper, a robust underwater vehicle sliding mode controller with fuzzy logic rules and root locus technique (FRSMC) is proposed. The systems considered are assumed to be completely state controllable. The control law consists of a continuous nominal control part and a discontinuous switching control part. The nominal part is determined using the nominal values of the system parameters, while the switching part deals with the parameter variations and disturbances. The well-known root locus technique [11] is a graphical method convenient for stability analysis and transient response design. It will be incorporated with the sliding mode control design to determine the continuous nominal control input. Exploiting the root locus technique in the design of sliding mode control makes possible the complete pole assignment [7]. Further, the fuzzy logic technique $[1,17]$ is employed to determine the discontinuous control input. The control law is developed based on collecting 


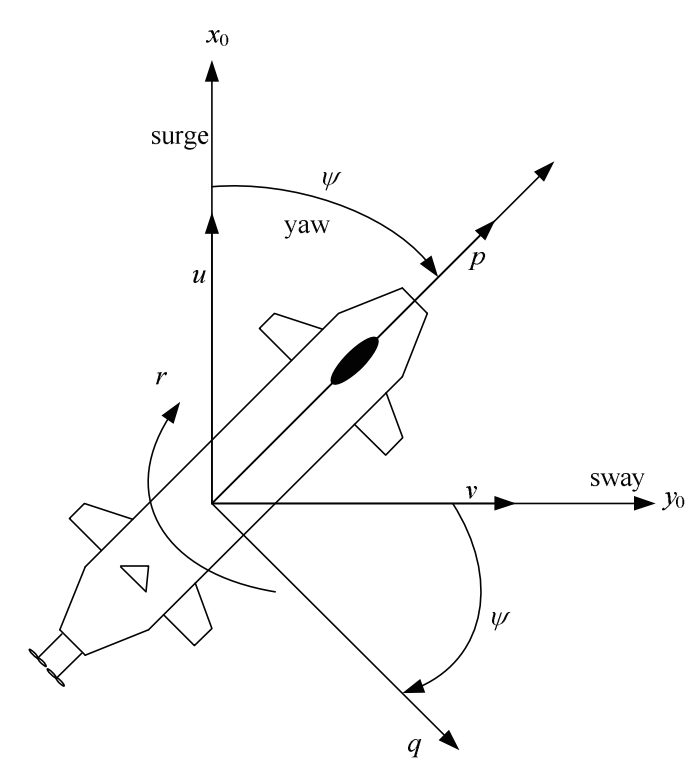

Fig. 1. Rotation over heading angle $\psi$ about $z_{0}$ while $w$ is fixed.

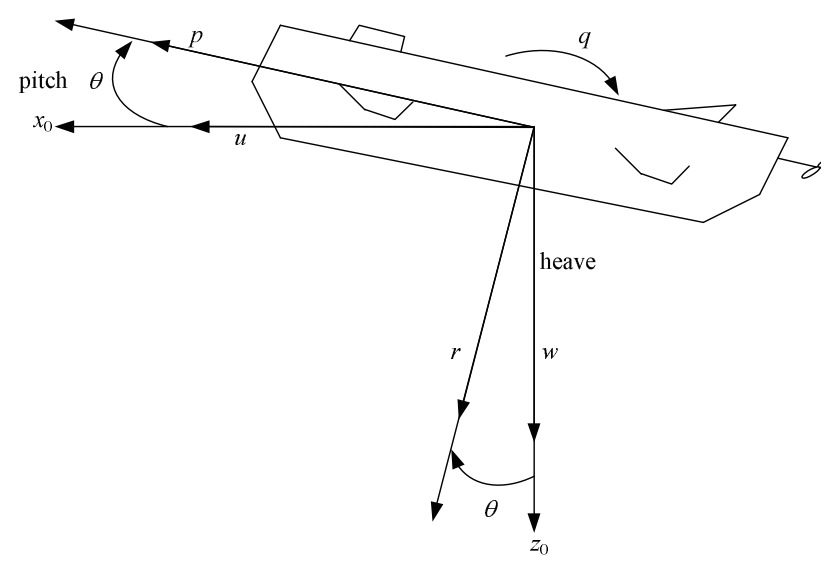

Fig. 2. Rotation over pitch angle $\theta$ about $y_{0}$ while $v$ is fixed.

human knowledge and is to deal with uncertainties in the control process.

In this study, application to the steering and diving control systems of underwater vehicles is carried out. It will be shown that system stability and robustness are achieved effectively.

\section{SYSTEM DYNAMICS DESCRIPTION}

Motion analysis in an underwater vehicle involves six degrees of freedom (DOF) as shown in Figs. 1-3 [4, 16]. Six independent coordinates are required to determine the position and orientation of a three-dimensional rigid body. The first three coordinates and their time derivatives correspond to the position and translational motion along the $x_{0}-, y_{0}-$, and $z_{0}$-axes, while the other three coordinates and their time derivatives are used to describe the orientation and rotational motion along the $p$-, $q$-, and $r$-axes. These six different motional components

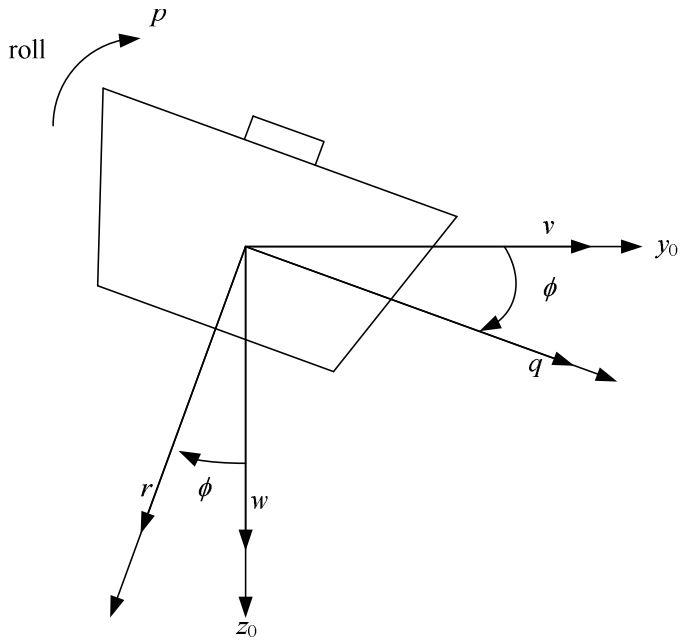

Fig. 3. Rotation over roll angle $\phi$ about $x_{0}$ while $u$ is fixed.

are conveniently defined as: surge, sway, heave, roll, pitch and yaw. Two non-interacting subsystems, steering and diving control systems, are considered.

The linearized dynamic equation for the steering control system of the considered underwater vehicle is given as

$$
\begin{aligned}
{\left[\begin{array}{c}
\dot{v} \\
\dot{r} \\
\dot{\psi}
\end{array}\right]=} & {\left[\begin{array}{ccc}
-0.27 \eta & 0.4 \eta & 0 \\
-0.05 \eta & -0.54 \eta & 0 \\
0 & 1 & 0
\end{array}\right]\left[\begin{array}{c}
v \\
r \\
\psi
\end{array}\right]+\left[\begin{array}{c}
0.12 \\
-0.15 \\
0
\end{array}\right] \delta_{r} } \\
& -\left[\begin{array}{c}
-0.27 \eta \\
-0.05 \eta \\
0
\end{array}\right] V_{c} \sin \left(\frac{\pi}{2}-\psi\right),
\end{aligned}
$$

where $v$ is the sway velocity, $r$ is the angular velocity in yaw, $\psi$ is the heading angle, and $\delta_{r}$ is the rudder deflection. The variable $\eta$ denotes the ratio of the longitudinal speed of the vehicle to a reference speed, and is supposed to be $\eta \in[1,3]$. Additionally, suppose the speed of the unknown ocean current can be represented as a sinusoidal function with amplitude of $V_{c}$ and phase angle of $\frac{\pi}{2}-\psi$. As seen in (1), it is obvious that the parametric variation in the system matrix and external multiplicative disturbances have great effect on the steering system performance. The aim here is to find a suitable controller such that the heading angle $\psi$ tracks the desired path $\psi_{d}$.

Next, a linearized diving system dynamics is considered. The diving system involves the pitch and the heave controls. The influence of the heave is assumed small and is omitted [5]. Therefore, the diving equations of motion include the pitch angular velocity $q$, the pitch angle $\theta$, the heave position $z$, and the stern plane deflection $\delta_{s}$. The linearized diving system dynamics is given as 


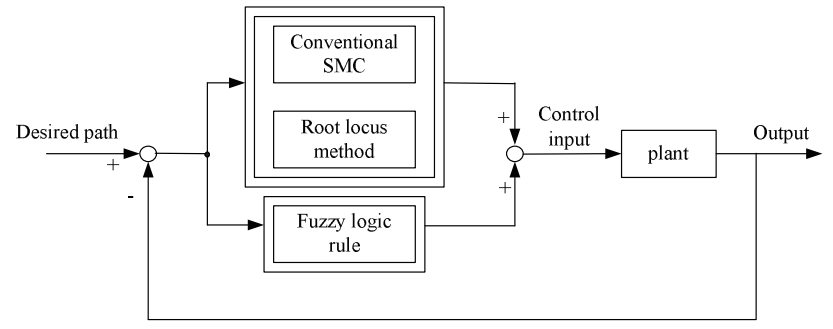

Fig. 4. Control system structure of FRSMC.

$$
\begin{gathered}
{\left[\begin{array}{c}
\dot{q} \\
\dot{\theta} \\
\dot{z}
\end{array}\right]=\left[\begin{array}{ccc}
-0.7+\Delta a_{11} & -0.3 & 0 \\
1 & 0 & 0 \\
0 & -1.832 \eta & 0
\end{array}\right]\left[\begin{array}{l}
q \\
\theta \\
z
\end{array}\right]+\left[\begin{array}{c}
0.035 \\
0 \\
0
\end{array}\right] \delta_{s}} \\
+\left[\begin{array}{c}
0.05 \\
0 \\
0
\end{array}\right] d .
\end{gathered}
$$

The variable $\eta$ is the same as in (1). The maximum value of the variation of the uncertain parameter $\Delta a_{11}$ is assumed to be 0.1 . The term $d$ denotes the external disturbance due to model imperfection and varying ocean current.

\section{FRSMC DESIGN}

In this section, a systematic design procedure of FRSMC for the steering control system (1) is demonstrated. A similar procedure is carried out for the diving control system (2) in the next section.

\section{Sliding Function Definition}

Let the steering control system (1) be represented in the general form as

$$
\begin{gathered}
\dot{\mathbf{x}}=\mathbf{A} \mathbf{x}+\mathbf{b} \delta_{r}+\mathbf{w}, \\
\mathbf{y}=\mathbf{c}^{T} \mathbf{x},
\end{gathered}
$$

where $x=\left[\begin{array}{lll}v & r & \psi\end{array}\right], b=\left[\begin{array}{lll}0.12 & -0.15 & 0\end{array}\right]^{T}, \mathbf{c}=\left[\begin{array}{lll}0 & 0 & 1\end{array}\right]^{T}$, and $\mathbf{w}$ is the disturbance denoting the last term in (1). The FRSMC control system structure is shown in Fig. 4. Since robustness is the concerned, the plant coefficient matrix is managed to be $\mathbf{A}=\mathbf{A}_{o}+\Delta \mathbf{A}$, where ' $。$ ' stands for the nominal part and ' $\Delta$ ' stands for the uncertain part. Select the nominal value of $\eta$ to be 2, i.e., $A_{o}=\left[\begin{array}{ccc}-0.54 & 0.8 & 0 \\ -0.1 & -1.08 & 0 \\ 0 & 1 & 0\end{array}\right]$. Then the nominal transfer function of (3) can be written as $G_{P}(s)=\frac{-0.15(s+0.62)}{s\left(s^{2}+1.62 s+0.6632\right)}=\frac{z(s)}{p(s)}$. The FRSMC design procedure involves two steps. First, the sliding function and the nominal control are determined by incorporating the root locus technique and the conventional SMC design. Second, the switching control law is tuned to deal with the parametric uncertainties and external disturbances by exploiting the fuzzy logic rules.

Conventionally, the sliding function is defined as $\sigma=\mathbf{g}^{T} \mathbf{x}$. Once the control system enters the sliding mode, the linear equivalent closed-loop system is obtained by setting $\dot{\sigma}$ to zero. The resulting system matrix is

$$
\hat{\mathbf{A}}_{e q}=\mathbf{A}-\mathbf{b}\left(\mathbf{g}^{T} \mathbf{b}\right)^{-1} \mathbf{g}^{T} \mathbf{A} .
$$

It is well known that one of the eigenvalues of $\hat{\mathbf{A}}_{e q}$ in (4) must be inevitably at the origin [17].

Placing all the closed-loop poles, not just a part of them, in the sliding mode for the nominal system in (3a) is the objective here. Let the heading angle error be $e(t)=\psi-\psi_{d}$. The goal is to achieve $e(\infty) \rightarrow 0$.

The sliding function is defined as

$$
\sigma=\mathbf{g}^{T}\left[\mathbf{x}-\mathbf{x}_{0}\right]+\lambda \int_{0}^{t} e(\tau) d \tau,
$$

where $\mathbf{x}_{0}$ is the initial condition of $\mathbf{x}$. The coefficient vector $\mathbf{g}$ and the number $\lambda$ are chosen to satisfy the closed-loop pole specification, and will be formulated later. In FRSMC, as defined in (5), $\sigma$ is forced to be zero since the beginning. Thus, the control system can reach sliding mode at once. The reaching time is effectively reduced.

Referring to the linear equivalent control, setting $\dot{\sigma}$ to zero, the equivalent control input is obtained as

$$
\delta_{r_{e q}}=-\left(\mathbf{g}^{T} \mathbf{b}\right)^{-1}\left(\mathbf{g}^{T} \mathbf{A} \mathbf{x}+\lambda\left(\psi-\psi_{d}\right)+\mathbf{g}^{T} \mathbf{w}\right) .
$$

Substituting (6) into system (3), the closed-loop state equation turns out to be

$$
\dot{\mathbf{x}}=\mathbf{A}_{e q} \mathbf{x}+\lambda \mathbf{b}\left(\mathbf{g}^{T} \mathbf{b}\right)^{-1} \psi_{d},
$$

where

$$
\mathbf{A}_{e q}=\mathbf{A}-\mathbf{b}\left(\mathbf{g}^{T} \mathbf{b}\right)^{-1}\left(\mathbf{g}^{T} \mathbf{A}+\lambda c^{T}\right)
$$

The closed-loop system poles in the sliding mode using FRSMC are identical to the eigenvalues of $\mathbf{A}_{e q}$ in (8) while the conventional SMC possesses the closed-loop system poles identical to the eigenvalues of $\hat{\mathbf{A}}_{e q}$ in (4).

Denote the characteristic polynomial of $\hat{\mathbf{A}}_{e q}$ as $\hat{p}(s)$ and zero polynomial of (3) as $z(s)$. Since one pole appears at the origin for the conventional SMC, we may define an equivalent open-loop transfer function.

$$
G(s)=\frac{\hat{k} z(s)}{\hat{p}(s)}=\frac{k\left(s+z_{1}\right)\left(s+z_{2}\right) \cdots\left(s+z_{l}\right)}{s\left(s+p_{1}\right)\left(s+p_{2}\right) \cdots\left(s+p_{n-1}\right)},
$$


where $l$ is the order of $z(s)$, and $n$ is the order of $\hat{p}(s)$. Briefly speaking, with the selection of sliding function (5) for system (3), the eigenvalues of $\mathbf{A}_{e q}$ in (8) are the same as the closed-loop poles of a unity feedback system whose forward transfer function is (9).

The aim here is to drive the control system to perform like a standard second order system. Note that a stable control system can be characterized by a pair of dominant poles if all other poles are 10 times further to the left than the dominant poles in the left half $s$-plane. The desired characteristics of transient and steady state responses can be obtained if the pair of dominant poles is appropriately chosen. The coefficient of the highest order of $s$ in $z(s)$ is in fact not necessarily one, therefore, $k$ is not equal to $\hat{k}$ in general.

Without loss of generality, assume that $n-1>l$. In the case of $n-1 \leq l$, the prefiltering technique [8] can be utilized to increase the relative degree and follows the same design procedure. The design procedure for the choice of $p_{i}$, $i=1,2, \cdots, n-1, \mathbf{g}$ and $h$ for constructing the sliding function is organized as follows:

Step 1. Let $p_{n-l}, p_{n-1-l}, \cdots, p_{n-1}$ be chosen equal to $z_{i}$ $i=1,2, \cdots, l$, respectively.

Remark: The open-loop transfer function of steering control system is $\quad G(s)=\frac{-0.15 \hat{k}(s+0.62)}{s\left(s+p_{1}\right)\left(s+p_{2}\right)}=\frac{k(s+0.62)}{s\left(s+p_{1}\right)\left(s+p_{2}\right)}$. Therefore, $p_{2}=0.62$.

Step 2. After pole-zero cancellation, the open-loop transfer function $G(s)$ becomes

$$
G_{1}(s)=\frac{k}{s\left(s+p_{1}\right)\left(s+p_{2}\right) \cdots\left(s+p_{n-1-l}\right)} .
$$

Let $s=s_{d 1}$ and $s=s_{d 2}$ be the required pair of the complex dominant poles according to the response specification. For $n-l=2$, proceed to Step 3. For $n-l \geq 3$, place the poles $-p_{2}$, $-p_{3}, \cdots,-p_{n-1-l}$ at least 10 times further than the dominant poles to the left of the imaginary axis in the $s$-plane.

Remark: The transfer function is $G_{1}(s)=\frac{k}{s\left(s+p_{1}\right)}$ after pole-zero cancellation procedure. Suppose the desired damping ratio and natural frequency are 0.95 and 7 , respectively. Then, the desired dominant poles are $s_{d 1}=-6.65+j 2.18$ and $s_{d 2}=-6.65-j 2.18$.

Step 3. Let the last un-specified pole $-p_{1}$ in Step 2 be determined by satisfying the following condition: $\angle G_{1}\left(s_{d 1}\right)$ equals to $(2 q+1) \pi$ for $k>0$ and $2 q \pi$ for $k<0, q=0$, $\pm 1, \pm 2, \cdots$.
Remark: $\left.\angle G_{1}(s)\right|_{s=-6.65+j 2.18}=-180^{\circ} \Rightarrow p_{1}=13.3$.

Step 4. The gain constant $k$ can be determined by applying $k=\left|\frac{1}{G(s)}\right|_{s=s_{d 1}}$. Then $\hat{k}$ can be obtained accordingly by dividing $k$ with the coefficient of the highest order of $\mathrm{s}$ in $z(s)$, as seen in (30).

Remark: $\quad k=|s(s+13.3)|_{s=-6.65+j 2.18}=48.9$, and hence $\hat{k}=48.9 /(-0.15)=-326$.

Step 5. Write $\hat{p}(s)=s^{n}+\hat{a}_{n-1} s^{n-1}+\hat{a}_{n-2} s^{n-2}+\cdots+\hat{a}_{1} s$ and $\operatorname{det}\left(s \mathbf{I}-\mathbf{A}_{o}\right)=s^{n}+a_{n-1} s^{n-1}+a_{n-2} s^{n-2}+\cdots+a_{1} s+a_{0}$. Define $\overline{\mathbf{p}}=\left[\begin{array}{llll}\hat{a}_{1} & \hat{a}_{2} & \cdots & \hat{a}_{n-1} 1\end{array}\right]^{T}, \quad \overline{\mathbf{C}}=\left[\begin{array}{llll}\mathbf{b} & \mathbf{A}_{o} \mathbf{b} & \cdots & \mathbf{A}_{o}^{n-1} \mathbf{b}\end{array}\right], \quad$ and $\overline{\mathbf{D}}=\left[\begin{array}{ccccc}a_{1} & a_{2} & \cdots & a_{n-1} & 1 \\ a_{2} & a_{3} & \cdots & 1 & 0 \\ \vdots & \vdots & \cdots & \vdots & \vdots \\ a_{n-1} & 1 & \cdots & 0 & 0 \\ 1 & 0 & \cdots & 0 & 0\end{array}\right]$. Then, $\mathbf{g}^{T}$ in $(7)$ can be determined by $\mathbf{g}^{T}=\overline{\mathbf{p}}^{T} \overline{\mathbf{D}}^{-1} \overline{\mathbf{C}}^{-1}$.

Remark: $\quad \overline{\mathbf{p}}^{T}=\left[\begin{array}{lll}8.25 & 13.9 & 1\end{array}\right] \quad, \quad$ and hence $\mathbf{g}^{T}=$ $\left[\begin{array}{lll}0 & -6.66 & -88.7\end{array}\right]$.

Step 6. Choose $h$ in (7) to be $h=\hat{k}\left(g^{T} b\right)^{-1}$.

Remark: For Case 1, $h=\hat{k}\left(g^{T} b\right)^{-1}=-326$.

In short, with closed-loop transfer function $T(s)=\frac{\hat{k} z(s)}{\hat{p}(s)+\hat{k} z(s)}$, the open-loop pole polynomial $\hat{p}(s)$ and the corresponding control gain $\hat{k}$ can be chosen to place all the closed-loop system poles. It is interesting to note that the polynomial $\hat{p}(s)$, exactly the closed-loop polynomial of the conventional sliding mode control, has inevitably one eigenvalue at the origin. The rest of eigenvalues of $\hat{p}(s)$ can be placed by suitably selecting the vector $\mathbf{g}$ in (5).

\section{FRSMC Control Law Formulation}

The control law must satisfy the sliding condition, $\sigma \dot{\sigma}<0$ in order that sliding mode exists and sustains. Since the vector $\mathbf{g}$ in (5) is chosen such that $\mathbf{g}^{T} \mathbf{b} \neq 0$ holds for all time, let the control input consist of a linear control $u_{o}$ and a switching control $u_{s}$, i.e.,

$$
u=u_{o}+u_{s}
$$

where 
Table 1. The fuzzy sets of $G, D G$ and $D K$.

\begin{tabular}{|c|c|c|}
\hline$G$ & $D G$ & $D K$ \\
\hline $\begin{array}{c}\text { B } \\
\text { (big) }\end{array}$ & $\begin{array}{c}\text { FL } \\
\text { (fast leaving) }\end{array}$ & $\begin{array}{c}\text { PB } \\
\text { (positive big) }\end{array}$ \\
\hline $\begin{array}{c}\text { M } \\
\text { (medium) }\end{array}$ & $\begin{array}{c}\text { SL } \\
\text { (slow leaving) }\end{array}$ & $\begin{array}{c}\text { PM } \\
\text { (positive medium) }\end{array}$ \\
\hline $\begin{array}{c}\text { S } \\
\text { (small) }\end{array}$ & $\begin{array}{c}\text { ZM } \\
\text { (zero movement) }\end{array}$ & $\begin{array}{c}\text { PS } \\
\text { (positive small) }\end{array}$ \\
\hline $\begin{array}{c}\text { Z } \\
\text { (zero) }\end{array}$ & $\begin{array}{c}\text { SA } \\
\text { (slow approaching) }\end{array}$ & $\begin{array}{c}\text { ZE (zero) } \\
\text { FA }\end{array}$ \\
\hline & $\begin{array}{c}\text { FA } \\
\text { (fast approaching) }\end{array}$ & $\begin{array}{c}\text { NM } \\
\text { (negative small) }\end{array}$ \\
\hline & & \\
\hline
\end{tabular}

$$
\begin{gathered}
u_{o}=-\left(\mathbf{g}^{T} \mathbf{b}\right)^{-1}\left(\mathbf{g}^{T} \mathbf{A}_{o} \mathbf{x}+\lambda y-\lambda y_{d}\right), \\
u_{s}=-\left|\mathbf{g}^{T} \mathbf{b}\right|^{-1}\left(\sum_{i=1}^{n} \alpha_{i}\left|x_{i}\right|+\alpha_{w}+\alpha_{0}\right) \operatorname{sgn}\left(\mathbf{g}^{T} \mathbf{b}\right) \operatorname{sgn}(\sigma) .
\end{gathered}
$$

The gain constants can be designed to be $\alpha_{i} \geq \sup \left|\left(\mathbf{g}^{T} \Delta \mathbf{A}\right)_{i}\right|$, $i=1,2, \cdots, n, \alpha_{w} \geq \sup \left|\mathbf{g}^{T} \mathbf{w}\right|, \alpha_{0} \geq 0$, and $\operatorname{sgn}(\zeta)$ equals 1 for $\zeta>0$ and -1 for $\zeta<0$. The notations $\left(\mathbf{g}^{T} \Delta \mathbf{A}\right)_{i}$ and $x_{i}$ stand for the $i$ th element of $\mathbf{g}^{T} \Delta \mathbf{A}$ and $\boldsymbol{x}$, respectively. It can be easily verified that $\dot{\sigma}<-\alpha_{0}$ for $\sigma>0$ and $\dot{\sigma}>\alpha_{0}$, for $\sigma<0$

Referring to the control structure in Fig. 4, the equivalent open-loop pole polynomial, $\hat{p}(s)$, can first be selected using the root locus technique. The vector $g$ can be determined to place arbitrarily the eigenvalues of $\hat{p}(s)$, except one at the origin. Then the gain constants, $\hat{k}$ and hence $\lambda=\hat{k}\left(\mathbf{g}^{T} \mathbf{b}\right)$, can be determined to place all the eigenvalues of the desired closed-loop pole polynomial, $d(s)$. Finally, with sliding function definition (5) and control laws (11)-(13), the control system is guaranteed to reach and stay in the sliding mode.

Next, consider that the bounds of the parameter variations and the external disturbances in (3) are not exactly known. The control parameters $\alpha_{i}$ and $\alpha_{w}$ may be not easily obtained. A fuzzy-logic-based switching law is to modify the above switching control component. Four principle components are involved in the fuzzy logic system in FRSMC. They are fuzzification interface, fuzzy rule base, fuzzy inference machine, and defuzzification interface.

Let $G, D G$ and $D K$ be linguistic variables representing two process variables, $|\sigma|$ and $\Delta|\sigma|$, and one control variable, $\Delta k$, respectively. The notations $\Delta|\sigma|$ and $\Delta k$ are the incremental changes of $|\sigma|$ and $k$, respectively. The fuzzy rules are given in

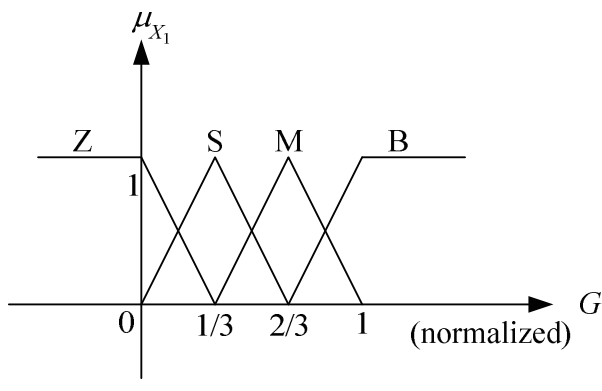

(a)

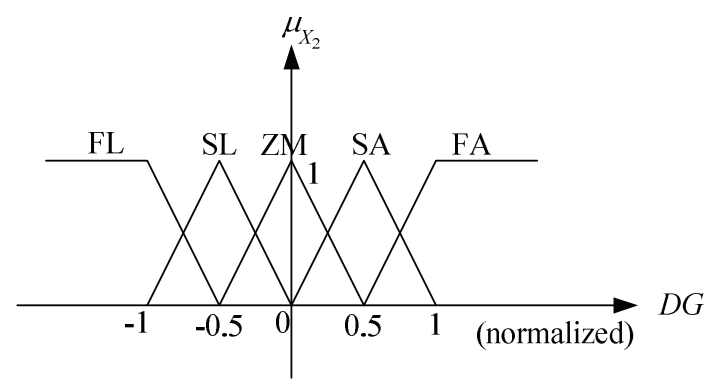

(b)

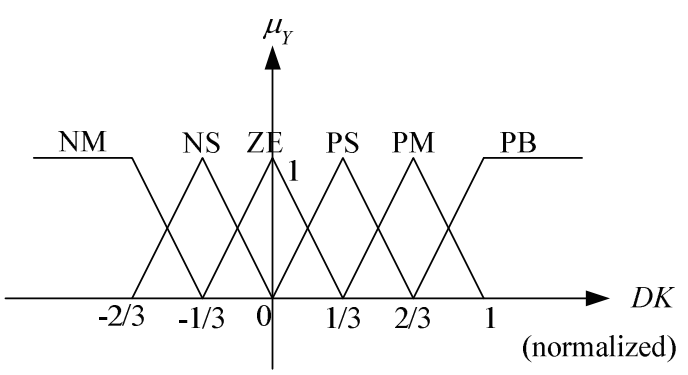

(c)

Fig. 5. (a) The membership function for $|\sigma|$, (b) The membership function for $\Delta|\sigma|$, (c) The membership function for $\Delta k(m T)$.

the form:

$$
R_{j} \text { : IF } G \text { is } X_{1}^{j} \text { and } D G \text { is } X_{2}^{j} \text { THEN } D K \text { is } Y^{j},
$$

where $X_{1}^{j}$ and $X_{2}^{j}$ are the fuzzy sets in the antecedent part, and $Y^{j}$ is the fuzzy set in the consequent part. The fuzzy sets of $G, D G$, and $D K$ are listed in Table 1 .

The membership functions used in a fuzzy logic control are usually parametric functions such as triangular functions, trapezoidal functions, and bell-shaped functions (Gaussian functions). In order to simplify the computer program, we choose the triangular-shaped membership functions. The membership functions of these two inputs variables and one output variable are defined with triangular-shaped functions, respectively, as shown in Fig. 5, where the universe of discourse of each input and output is normalized over the interval $[-1,1]$. The membership functions are equally distributed in the universe discourse. We use mainly the AND operation. The evaluation of rule $R_{j}$ results in a minimum of $\mu_{X_{1}^{j}}$ and $\mu_{X_{2}^{j}}$, 
Table 2. The fuzzy rule base.

\begin{tabular}{|c|c|c|c|c|c|}
\hline 1. GG & FL & SL & ZM & SA & FA \\
\hline B & PB & PM & PM & PM & PS \\
\hline M & PB & PM & PM & PS & ZE \\
\hline S & PM & PS & PS & ZE & NS \\
\hline$Z$ & PS & PS & ZE & NS & NM \\
\hline
\end{tabular}

i.e.,

$$
w^{j}=\min \left\{\mu_{X_{1}^{j}}, \mu_{X_{2}^{j}}\right\},
$$

where $w^{j}$ is the degree of matching of each rule in the antecedent part, $\mu_{X_{1}^{j}}$ and $\mu_{X_{2}^{j}}$ are the membership grades of the two distinct inputs in rule $R_{j}$, respectively. Next, a fuzzy inference engine is set up to perform the decision making logic. Some of the fuzzy rules in the fuzzy rule base will be fired to determine the weighted output of the fuzzy logic system. Using the min-product-max inference method $[12,13]$ to obtain the degree of consequence yields

$$
Y^{j}=w^{j} \cdot \mu_{Y^{j}},
$$

where $\mu_{Y^{j}}$ is the membership grade of the output in rule $R_{j}$.

Let $T$ be the sampling time and $\Delta k(m T)$ be the crisp controller output at the $m$ th sample. The crisp output is obtained by using the center of gravity defuzzification method as

$$
\Delta k(m T)=\frac{\sum_{j=1}^{N} w^{j} Y^{j}}{\sum_{j=1}^{N} w^{j}},
$$

where $N$ is the number of rules, $w^{j}$ is the degree of matching of each rule in the antecedent part, and $Y^{j}$ represents the inference value in the consequent part of the fuzzy rule $j$. Consequently, the switching control gain $k(m T)$ is formulated as

$$
k(m T)=k((m-1) T)+\Delta k(m T) .
$$

Thus, the switching control gain is designed as

$$
u_{s}(m T)=-k(m T) \operatorname{sgn}\left(g^{T} b\right) \operatorname{sgn}(\sigma) .
$$

The principle of the fuzzy logic here can be briefly stated as follows. If the system trajectory is far from the sliding surface, then the switching control gain is increased to make the system trajectory move faster toward the sliding surface. Alternatively, if the system trajectory is near the vicinity of the sliding surface, a small switching control gain is needed, and thus the control gain is decreased.

\section{NUMERICAL RESULT}

\section{FRSMC for Steering Control System}

The dynamic equation for the steering control system (1) is considered. The desired output path is assigned as follows:

$$
y_{d}=\left\{\begin{array}{cc}
0.785 \sin \left(\frac{\pi}{35} t+1.5 \pi\right)+0.785, & \text { for } \quad 0 \leq t \leq 35, \\
0.5 \pi, & \text { for } t>35 .
\end{array}\right.
$$

Selecting properly the dominant pole positions is important. Note that overshoot is a function of the damping ratio and will get smaller when the damping ratio is closer to 1 . In addition, the response speed is a function of the natural undamped frequency and will get faster if the natural undamped frequency gets larger. Here, the damping ratio is selected to be 0.95 and the natural undamped frequency is selected to be 7 . The equivalent open-loop transfer function, $G(s)$, is given $(\eta=2)$ as

$$
G(s)=\frac{-0.15 \hat{k}(s+0.62)}{\hat{p}(s)}=\frac{K(s+0.62)}{s\left(s+p_{1}\right)\left(s+p_{2}\right)} .
$$

Following the design procedure described in Step 1 to Step 6, choose $p_{2}=0.62, p_{1}=13.3$, and $\hat{k}=-326$. For the desired dominant pole positions been specified at $-6.65 \pm j 2.18$, $\mathbf{g}=\left[\begin{array}{lll}0 & -6.66 & -88.7\end{array}\right]^{T}$ and $\lambda=-326$. Consequently, the sliding function can be obtained as

$$
\sigma=\left[\begin{array}{lll}
0 & -6.66 & -88.7
\end{array}\right]\left(\mathbf{x}-\mathbf{x}_{0}\right)-326 \int_{0}^{t}\left(y-y_{d}\right) d \tau .
$$

Combining the fuzzy logic rules yields the control input as

$$
u=\left[\begin{array}{lll}
-0.66 & 81.5 & 0
\end{array}\right] \mathbf{x}+326\left(y-y_{d}\right)-k(m T) \operatorname{sgn}(\sigma) .
$$

The discontinuous switching control input is determined using fuzzy logic control. The fuzzy rules are tabulated in Table 2 . Control rules in Table 2 are developed from the following concept: if the state trajectories are far away (or moving away) from the intersection of the sliding surfaces, then the amplitude of the discontinuous control gain should be large; on the contrary, the control gain is decreased when the state trajectories are near (or approaching) the intersection of the sliding surfaces. The membership functions of these two input variables and one output variable are shown in Fig. 5. Using the min-min-max inference method and the center of gravity method, the incremental change of the discontinuous control gain $\Delta k(m T)$ and $k(m T)=k((m-1) T)+\Delta k(m T)$ is obtained.

Figures 6 to 9 show the steering control system response. As shown in Fig. 6, the underwater vehicle can always track the expected heading angle path without being affected by the 


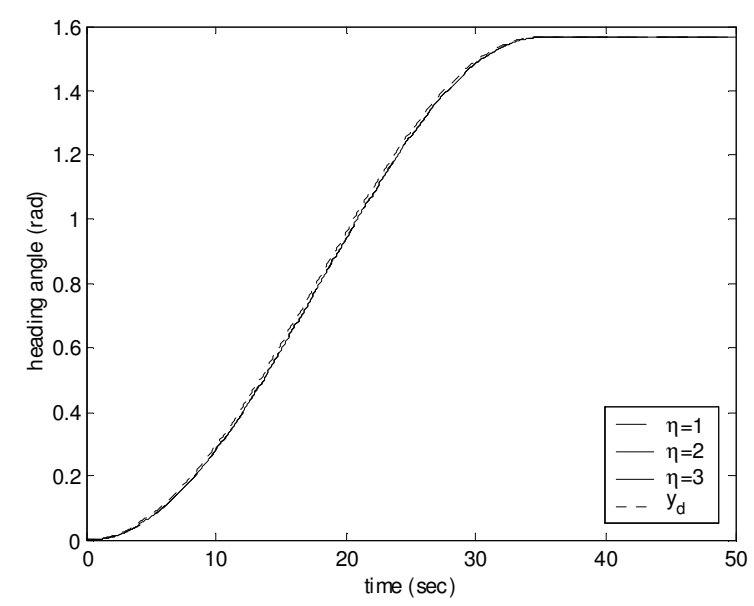

Fig. 6. The heading angle $\psi$ in steering.

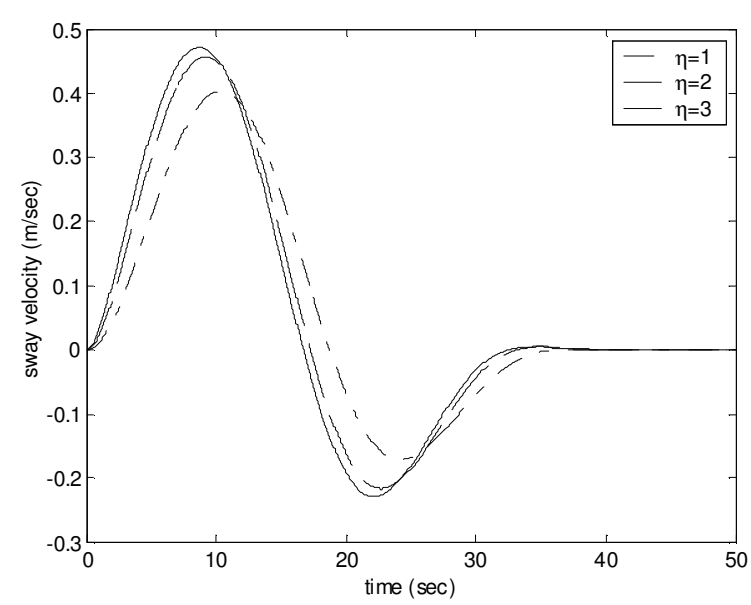

Fig. 7. The sway velocity $v$ in steering.

ocean currents and various longitudinal speeds. It takes only 35 seconds to turn the heading angle $\psi$ from 0 to $1.57 \mathrm{rad}$ without any output overshoot and the steady-state error is near zero. As seen in Figs. 7 and 8, the surge velocity and the yaw angular velocity are asymptotically stable no matter when the underwater vehicle is running at low or high speed. The FRSMC demonstrates its remarkable tracking robustness. For the control input, as shown in Fig. 9, more control effort is needed for the underwater vehicle traveling at high speed to have the same performance as traveling at low speed. It is also noted that, in order to have a satisfactory performance when making a course control, the underwater vehicle should not travel too fast in order to ensure that rudder deflection is controlled within a reasonable range.

\section{FRSMC for Diving Control System}

Let the diving control system (2) be represented in the general form as

$$
\dot{\mathbf{x}}=\mathbf{A} \mathbf{x}+\mathbf{b} \delta_{s}+\mathbf{w}
$$

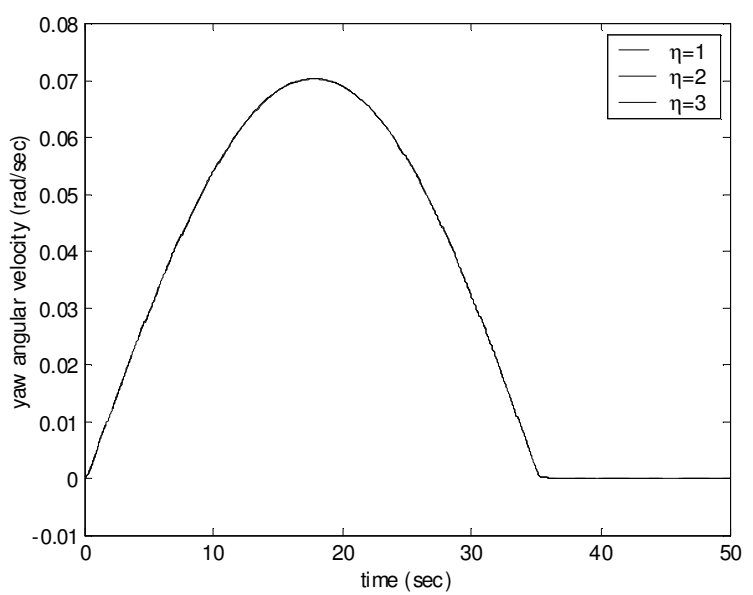

Fig. 8. The yaw angular velocity $r$ in steering.

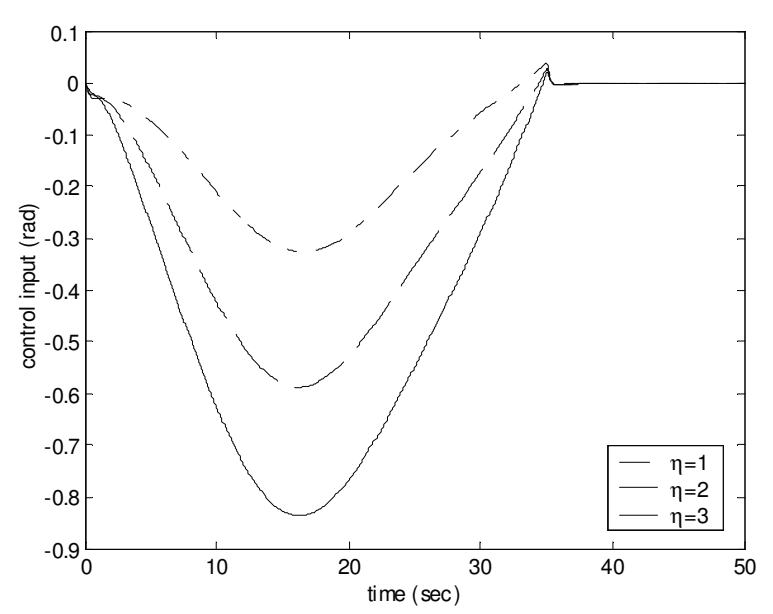

Fig. 9. The control input $\delta_{r}$ in steering.

$$
y=\mathbf{c}^{T} \mathbf{x},
$$

where $\mathbf{x}=\left[\begin{array}{lll}q & \theta & z\end{array}\right]^{T}, \quad \mathbf{b}=\left[\begin{array}{lll}0.035 & 0 & 0\end{array}\right]^{T}, \quad \mathbf{c}=\left[\begin{array}{lll}0 & 0 & 1\end{array}\right]^{T}$, and $\mathbf{w}$ is the disturbance denoting the last term in (2). The nominal plant coefficient matrix is $\mathbf{A}_{o}=\left[\begin{array}{ccc}-0.7 & -0.3 & 0 \\ 1 & 0 & 0 \\ 0 & -3.664 & 0\end{array}\right]$ for $\eta=2$. The nominal transfer function of diving control system is $G_{P}(s)=\frac{-0.12824}{s\left(s^{2}+0.7 s+0.3\right)}=\frac{z(s)}{p(s)}$. The initial value of the state variable is assumed to be $\mathbf{x}_{0}=\left[\begin{array}{lll}0 & 0 & 0\end{array}\right]^{T}$.

Let the second order reference model has damping ratio of 0.9 and natural undamped frequency of $0.5 \mathrm{rad} / \mathrm{s}$. Hence, the desired dominant pole positions are specified at $-0.45 \pm j 0.217$. Referring to Section 3.1, the sliding function is defined by the following steps:

Step 1. The open-loop transfer function of diving control 


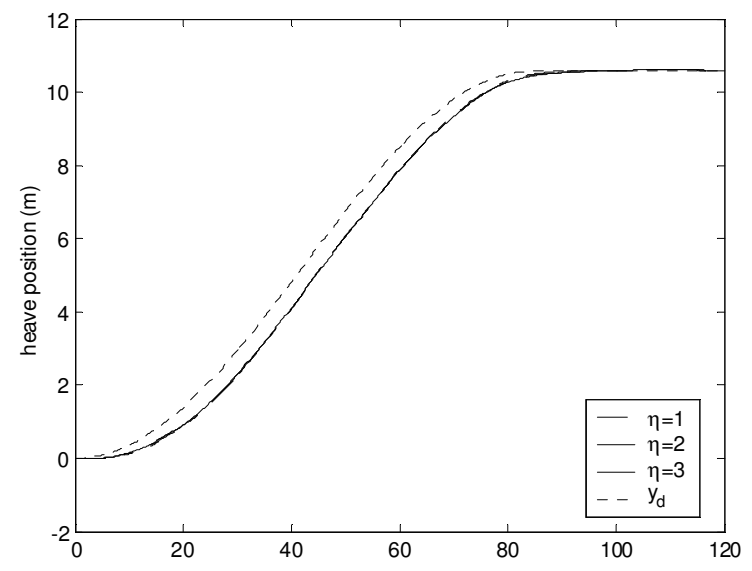

Fig. 10. The heave position (output) $z$ in diving.

system is $G(s)=\frac{-0.128 \hat{k}}{s\left(s+p_{1}\right)\left(s+p_{2}\right)}=\frac{k}{s\left(s+p_{1}\right)\left(s+p_{2}\right)}$. This control system does not possess any zero, so we need not choose any pole to cancel the zero.

Step 2. The transfer function is $G_{1}(s)=\frac{k}{s\left(s+p_{1}\right)\left(s+p_{2}\right)}$. Suppose the desired damping ratio and natural frequency are 0.9 and 0.5 , respectively. Then, the desired dominant poles are $s_{d 1}=-0.45+j 0.217$ and $s_{d 2}=-0.45-j 0.217$. Because this diving control system has three poles, let the third pole $-p_{2}=-4.5$, which is 10 times further to the left than the specified dominant poles in the LHS of $s$-plane.

Step 3. Applying $\left.\angle G_{1}(s)\right|_{s=-0.45+j 0.217}=-180^{\circ}$, the last un-specified pole $-p_{1}$ is found to be -0.96 .

Step 4. The gain constants are $k=|s(s+4.5)(s+0.92)|_{s=-0.45+j 0.217}=1.14$ and

$\hat{k}=1.14 /(-0.128)=-8.89$.

Step 5. Choose $\quad \overline{\mathbf{p}}^{T}=\left[\begin{array}{lll}4.32 & 5.46 & 1\end{array}\right] \quad$ and $\mathbf{g}^{T}=\left[\begin{array}{lll}28.6 & 156 & -34.0\end{array}\right]$.

Step 6. Apply $h=\hat{k}\left(g^{T} b\right)^{-1}=-8.89$.

Consequently, the resulting sliding function is

$$
\sigma=\left[\begin{array}{lll}
28.6 & 156 & -34.0
\end{array}\right]\left(\mathbf{x}-\mathbf{x}_{0}\right)-8.89 \int_{0}^{t}\left(y-y_{d}\right) d \tau .
$$

Suppose the desired output path $y_{d}$ is

$$
y_{d}= \begin{cases}5.3 \sin \left(\frac{\pi}{55} t+1.5 \pi\right)+5.3, & \text { for } 0 \leq t \leq 85, \\ 10.6, & \text { for } t>85 .\end{cases}
$$

Applying the design procedure in previous section yields

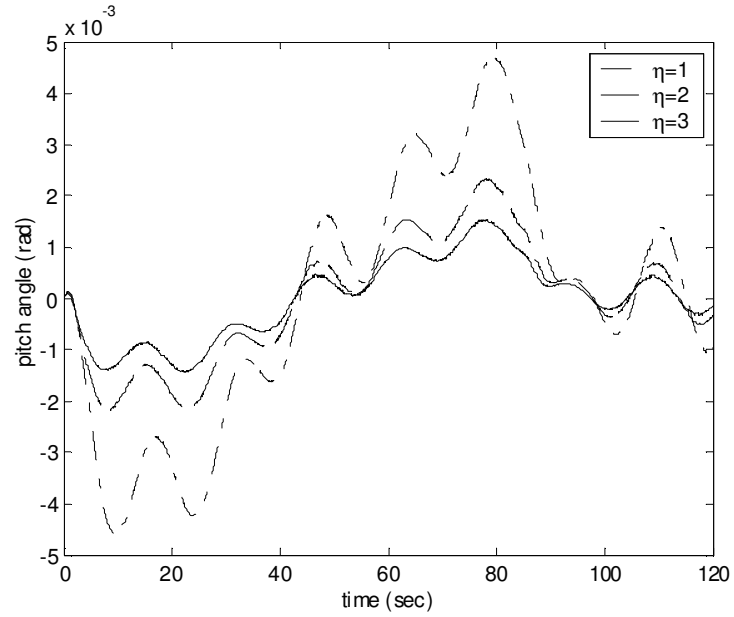

Fig. 11. The pitch angle $\theta$ in diving.

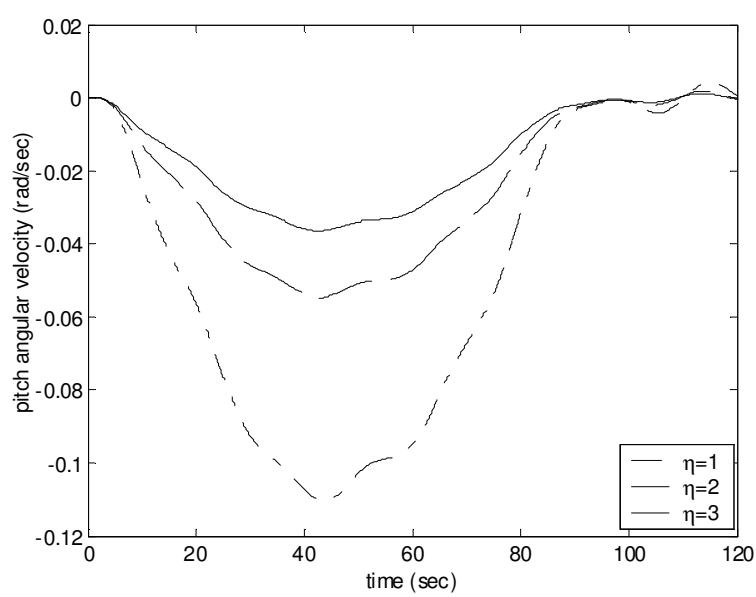

Fig. 12. The pitch angular velocity $q$ in diving.

$u=\left[\begin{array}{lll}-136 & -116 & 0\end{array}\right] \mathbf{x}+8.89\left(y-y_{d}\right)-k(m T) \operatorname{sgn}(\sigma)$.

Figures 10 to 14 show the diving control system responses. Figure 10 shows the output response for the underwater vehicle diving $10.6 \mathrm{~m}$. The FRSMC controller allows the underwater vehicle to dive smoothly and possess strong stability and robustness. Referring to Fig. 11, the underwater vehicle is tilted no more than $0.035 \mathrm{rad}$ or $0.105 \mathrm{rad}$ to accomplish the required tracking performance when traveling at a high or low speed, respectively. Hence, in order to reach the required diving depth as fast as possible, the underwater vehicle should travel at a higher speed. Fig. 12 shows the pitch angular velocity that tends to vanish after the diving task is completed. The control input, stern plane deflection, needs less effort when traveling at a high speed as shown in Fig. 13. When an external bounded disturbance occurs, as shown in Fig. 14, system robustness is maintained. The heave position performance and the pitch angle are robust against the external disturbance. The control activity demonstrates the disturbance rejection effort that leads to a smooth heave position tracking. Moreover, applying the boundary layer technique, the system chattering problem is 


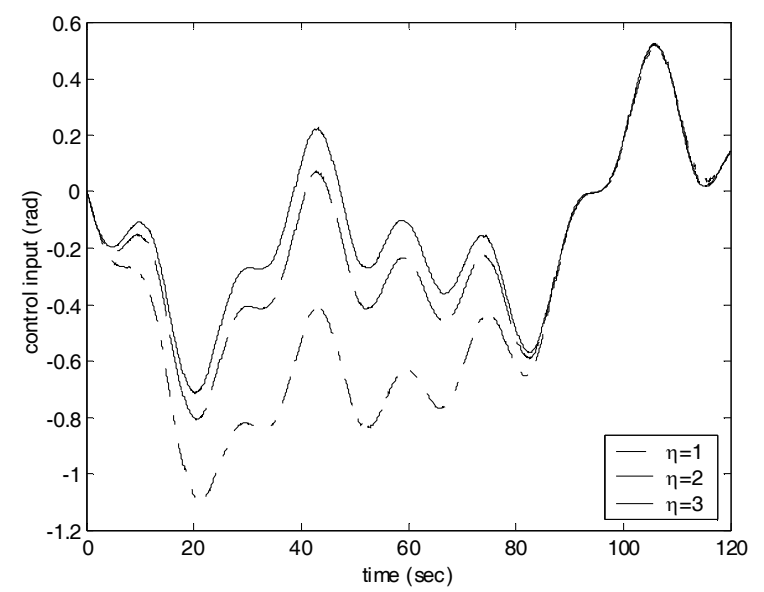

Fig. 13. The control input $\delta_{s}$ in diving.

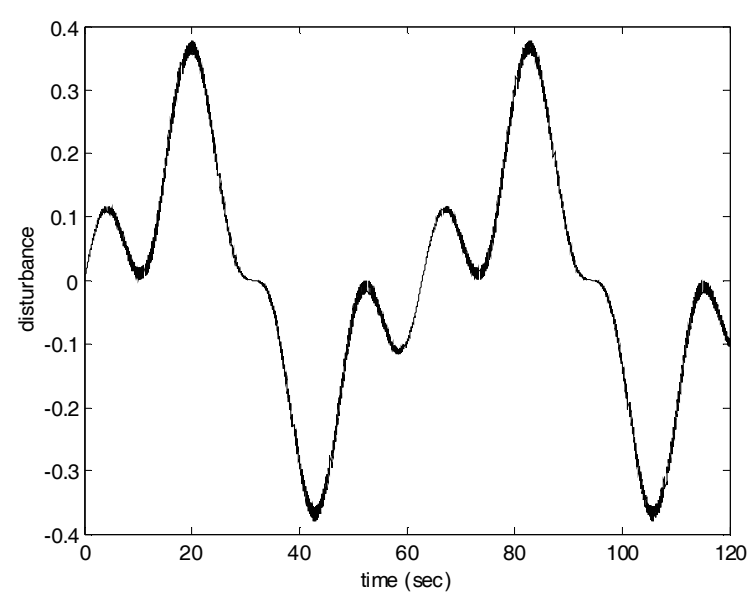

Fig. 14. The bounded external disturbance $d(t)$.

greatly eliminated.

In summary, as indicated in the above simulation results, though the pole-zero cancellation was not exactly executed for all various $\eta$, the proposed FRSMC is successfully applied to the underwater vehicle control systems and provides strongly robust system performance. Comparing with [2, 10, 18], the proposed controller focuses on sliding mode control which can place all the closed-loop poles in the sliding mode by using the sliding mode and root locus techniques. The fuzzy logic technique is employed to determine the discontinuous control input gain to reduce the input chattering. This paper presents the advantage of integrating the sliding mode control, root locus techniques, and fuzzy logic technique. In [2, 10, 18], these papers used the intelligent control to control the underwater vehicle and the intelligent control is the main controller. Those methods focused on deigning intelligent controller to obtain good performance. In this paper, the fuzzy logic is used to reduce the input chattering to support sliding mode control.

\section{CONCLUSION}

In this paper, a robust sliding mode control with root locus technique and fuzzy logic rules is successfully developed. Root locus technique has made possible a complete pole assignment that conventional sliding mode control could not accomplish. The control system is able to follow a standard second order reference model. Thus, steady state and transient response can be assigned. The fuzzy logic rules guarantees the robustness against parameter variations and external disturbance. The main advantages of the proposed FRSMC are: (1) control system possesses strong robustness, (2) steady-state error can be effectively eliminated, and (3) all closed-loop poles in the sliding mode can be freely allocated as long as the system is completely controllable. Future work is the extension of this work to multi-input multi-output systems.

\section{ACKNOWLEDGMENTS}

The authors would like to thank the National Science Council, Taiwan, R.O.C., for supporting this work under Grant NSC95-2221-E-231-013 and NSC95-2622-E-155-008 -CC3.

\section{REFERENCES}

1. Chang, Y. C., "Robust tracking control for nonlinear MIMO systems via fuzzy approaches," Automatica, Vol. 36, No. 10, pp. 1535-1545 (2000).

2. Craven, P. J., Sutton, R., Burns, R. S., and Dai, Y. M., "Multivariable intelligent control strategies for an autonomous underwater vehicle," International Journal of Systems Science, Vol. 30, No, 9, pp. 965-980 (1999).

3. Fossen, T. I., Guidance and Control of Ocean Vehicle, John Wiley \& Sons, New York (1994).

4. Fossen, T. I. and Blanke, M., "Nonlinear output feedback control of underwater vehicle propellers using feedback form estimated axial flow velocity," IEEE Journal of Oceanic Engineering, Vol. 25, No. 2, pp. 241-255 (2000).

5. Healey, A. J. and Lienard, D., "Multivariable sliding-mode control for autonomous diving and steering of unmanned underwater vehicles," IEEE Journal of Oceanic Engineering, Vol. 18, No. 3, pp. 327-339 (1993).

6. Huang, Y. J. and Way, H. K., "Design of sliding surfaces in variable structure control via a direct pole assignment scheme," International Journal of Systems Science, Vol. 32, No. 8, pp. 963-969 (2001).

7. Huang, Y. J. and Way, H. K., "Placing all closed loop poles of missile attitude control systems in the sliding mode via the root locus technique," ISA Transactions, Vol. 40, No. 4, pp. 333-340 (2001).

8. Huang, Y. J. and Yeung, K. S., "A control scheme robust against all parameter variations and disturbances," International Journal of Systems Science, Vol. 25, No. 10, pp. 1621-1629 (1994).

9. Hung, J. Y., Gao, W., and Hung, J. C., "Variable structure control: a survey," IEEE Transactions on Industrial Electronics, Vol. 40, No. 1, pp. 2-22 (1993).

10. Jagannathan, S. and Galan, G., "One-layer neural-network controller with preprocessed inputs for autonomous underwater vehicles," IEEE Transactions on Vehicular Technology, Vol. 52, No. 5, pp. 1342-1355 (2003).

11. Kuo, B. C. and Golnaraghi, F., Automatic Control Systems, 8th ed, John Wiley \& Sons, New York (2002).

12. Lee, C. C., "Fuzzy logic in control systems: fuzzy logic controller, part I," IEEE Transactions on Systems, Man, and Cybernetic, Vol. 20, No. 2, pp. 404-418 (1990).

13. Lee, C. C., "Fuzzy logic in control systems: fuzzy logic controller, part II,' IEEE Transactions on Systems, Man, and Cybernetic, Vol. 20, No. 2, pp. 419-435 (1990).

14. Lee, P. M., Hong, S. W., Lim, Y. K., Lee, C. M., Jeon, B. H., and Park, J. W., "Discrete-time quasi-sliding mode control of an autonomous underwater vehicle," IEEE Journal of Oceanic Engineering, Vol. 24, No. 3, pp. 
388-395 (1999)

15. Utkin, V. I., "Variable structure systems with sliding modes," IEEE Transactions on Automatic Control, Vol. 22, No. 2, pp. 212-222 (1977).

16. Valavanis, K. P. and Gracanin, D., "Control architectures for autonomous underwater vehicles," IEEE Control Systems Magazine, Vol. 17, No. 6, pp. 48-64 (1997).

17. Wang, H. O., Tanaka, K., and Griffin, M. F., "An approach to fuzzy control of nonlinear systems: stability and design issue," IEEE Transactions on Fuzzy Systems, Vol. 4, No. 1, pp. 14-23 (1996).
18. Wang, J. S. and Lee, C. S. G., "Self-adaptive recurrent neuro-fuzzy control of an autonomous underwater vehicle," IEEE Transactions on Robotics and Automation, Vol. 19, No. 2, pp. 283-295 (2003).

19. Young, K. D., Utkin, V. I., and Özgüner, Ü., “A control engineer's guide to sliding mode control," IEEE Transactions on Control Systems Technology, Vol. 7, No. 3, pp. 328-342 (1999).

20. Yuh, J., "Modeling and control of underwater robotic vehicles," IEEE Transactions on Systems, Man, and Cybernetic, Vol. 20, No. 6, pp. 1475-1483 (1990). 